\title{
The Number of Conjugacy Classes and Irreducible Characters in a Finite Group
}

\section{Remigius Okeke Aja ${ }^{1, *}$, Uchenna Emmanuel Obasi ${ }^{2}$ and Everestus Obinwanne Eze ${ }^{3}$}

${ }^{1}$ Department of Mathematics, College of Physical and Applied Science, Michael Okpara University of Agriculture, Umudike, Nigeria; e-mail: ajah.remigius@mouau.edu.ng

${ }^{2}$ Department of Mathematics, College of Physical and Applied Science, Michael Okpara University of Agriculture, Umudike, Nigeria; e-mail: sirurchobasi@ gmail.com

${ }^{3}$ Department of Mathematics, College of Physical and Applied Science, Michael Okpara University of Agriculture, Umudike, Nigeria; e-mail: obinwanneeze@gmail.com

* Corresponding author

\begin{abstract}
In this paper, number of conjugacy classes and irreducible characters in a non-abelian group of order $2^{6}$ are investigated using cycle pattern of elements. Through the exploits of commutator and representation of elements as a product of disjoint cycles, the number of conjugacy classes is obtained which extends some results in literature.
\end{abstract}

\section{Introduction}

The concept of conjugacy classes and irreducible characters which started since the time of Burnside (1852-1927) and Frobenius (1849-1917), at the present time plays a more important role in the study of groups and their representations. In recent time, the exploration of conjugacy classes has been the active subject and remains a major area of

Received: April 21, 2019; Accepted: May 31, 2019

2010 Mathematics Subject Classification: 34B15, 34C15, 34C25, 34K13.

Keywords and phrases: conjugacy classes, irreducible characters, non-abelian group, cycle pattern, cummutator.

Copyright (ㅇ 2019 Remigius Okeke Aja et al. This is an open access article distributed under the Creative Commons Attribution License, which permits unrestricted use, distribution, and reproduction in any medium, provided the original work is properly cited. 
attention for many researchers as observed in [1] and [2]. The important part of this group under discussion is the conjugacy classes of a non-abelian group of order sixty four $\left(2^{6}\right)$.

Let $G$ be a finite group. Two elements $a$ and $b$ of $G$ are conjugate if there exists $g \in G$ such that $\mathrm{gag}^{-1}=b$. Conjugacy classes of finite group $S_{n}$ can be represented using a cycle type which also shows the partition of $n$ as demonstrated in [3]. [4], [5] and [6] had worked on conjugacy classes of finite group with resounding results. Motivated by the above literature and on-going research in this direction, the objective of this paper is to find the number of conjugacy classes and irreducible characters in a finite group of non-abelian group of order $2^{6}$. Definitions and theorems corresponding to conjugacy classes are presented with proofs. Furthermore, conjugacy classes of a finite group together with their cycle pattern are presented using three subgroups of $G$, where $G$ is non-abelian.

\section{Preliminaries}

Definition 2.1. Let $G$ be a finite group. Then a conjugacy class $C(G)$ is a non-empty subset of $G$ such that the following holds:

(i) Given any $x, y \in C$ there exists $g \in G$ such that $\operatorname{gxg}^{-1}=y$.

(ii) If $x \in C$ and $g \in G$, then $g x g^{-1}=C$. In other words, it is closed under the action of group on itself.

Definition 2.2. Let $G$ be a finite group consisting of $n$ elements. Then a permutation group of degree $n$ is a one to one mapping of $S_{n}$ onto itself.

Definition 2.3. Let $G$ be a finite group. Then the group of all permutations of $G$ is the symmetric group on $n$ letter which is denoted by $S_{n}$.

Definition 2.4. An irreducible character is a representation that has no non-trivial invariant subspace.

Proposition 2.1. Any permutation of a finite set containing at least two elements can be written as the product of transpositions. 
Theorem 2.1. Every permutation in $S_{n}$ can be written as the product of disjoint cycles.

Proof. We can assume that $X=\{1,2, \ldots, n\}$. Let $\sigma \in S_{n}$ and $X_{1}=\left\{\sigma(1), \sigma^{2}(1), \ldots\right\}$. The set $X_{1}$ is finite since $X$ is finite. Now let $i$ be the first integer in $X$ that is not in $X_{1}$ and $X_{2}=\left\{\sigma(i), \sigma^{2}(i), \ldots\right\}$. Again $X_{2}$ is a finite set. Continuing in that manner, we can define finite disjoint sets $X_{3}, X_{4}, \ldots$. Since $X$ is finite set, we are guaranteed that this process will end and there will be only a finite number of these sets say $\gamma$. If $\sigma_{i}$ is the cycle defined by

$$
\sigma_{i}(x)=\left\{\begin{array}{ll}
\sigma(x) & x \in X_{i}, \\
x & x \notin X_{i},
\end{array} \quad \text { for } i=1,2, \ldots, r\right.
$$

then $\sigma=\sigma_{1}, \sigma_{2}, \ldots, \sigma_{r}$. Since the set $X_{1}, X_{2}, \ldots, X_{r}$ are disjoint, the cycles $\sigma_{1}, \sigma_{2}, \ldots, \sigma_{r}$ must be disjoint. See [7].

Theorem 2.2. Let $(G, o)$ be a group. Then, a conjugacy relation of $G$ is an equivalence relation on $G$.

Proof. Let $(\sim)$ be a conjugacy relation defined on $G$. Then it is required to show that $(\sim)$ is reflexive, symmetry and transitive. $\forall x \in G$ there exists $e_{G}$ such that

$$
e_{G} o x=x^{\circ o e_{G}} \Rightarrow x \sim x,
$$

where $e_{G}$ is the identity in $G$. Thus conjugacy relation of $G$ is reflexive. $\forall x, y \in G$ there exists $a \in G$ such that

$$
x \sim y \Rightarrow \text { aox }=\text { yoa } .
$$

Post multiply (1.3) by $a^{-1}$ yields

$$
\operatorname{aoxoa}^{-1}=y
$$

which implies that $y \sim x$. Thus conjugacy relation of $G$ is symmetric. $\forall x, y, z \in G$ there exist $a_{1}$ and $a_{2}$ such that

$$
\begin{aligned}
& x \sim y \Rightarrow a_{1} o x=y o a_{1} . \\
& y \sim z \Rightarrow a_{2} o y=z o a_{2} .
\end{aligned}
$$


From (1.5) we have

$$
a_{1} \text { oxoa }_{1}^{-1}=y \text {. }
$$

Putting (1.7) in (1.6) gives

$$
a_{2} o a_{1} o x o a_{1}^{-1}=z o a_{2}
$$

Post multiply (1.8) by $a_{1}$ gives

$$
\begin{gathered}
a_{2} o a_{1} o x o a_{1}^{-1} o a_{1}=z o a_{2} o a_{1}, \\
a_{2} o a_{1} o x e=z o a_{2} o a_{1}, \\
a_{2} o a_{1} o x=z o a_{2} o a_{1}
\end{gathered}
$$

which implies that $x \sim z$. Thus conjugacy relation of $G$ is transitive. Hence conjugacy relation is an equivalence relation.

Remark. Since conjugacy relation is an equivalence relation, it partitions elements of the group into equivalence classes. This means that every element of the group belongs precisely to one conjugacy class and classes $C \mid(x)$ and $C \mid(y)$ are equal if and only if $x$ and $y$ are conjugate and disjoint otherwise. The equivalence class that contains $x \in G$ is $C \mid(x)=\left\{g x g^{-1}: g \in G\right\}$ and is called the conjugacy class of $x$.

Definition 2.5. The commutator of two elements $w$ and $p$ of a group $G$ is the element $[w, p]=w^{-1} p^{-1} w p$ and it is equal to group identity if and only if $w$ and $p$ commute. The set of all commutators of a group is not in general closed under the group operation but the subgroup of $G$ generated by all commutators is closed and is called the commutator subgroup of $G$.

\section{Results}

\subsection{Conjugacy classes of $\boldsymbol{G}$ of order $2^{6}$}

We consider a non-abelian group $G=\{x, y\}$ with three subgroups $p, q, r$ of $G$ defined as $p=(12), q=(13)(24)$ and $r=(15)(26)(37)(48)$. Let $x=p q$ and $y=q r$ such that $p q \neq q r$. Representing $G$ as a product of disjoint cycles using some commutator identities and element of $G: x, y,[x, y],[x, y]^{2}, x y, x^{2}, y^{-1}, x[x, y]^{2}$, 
$(x y)^{2}, x y^{2}, y^{2}$ and $x y^{-1}$, we have

$$
\begin{aligned}
& \text { (i) } x=p q=\left(\begin{array}{lllllll}
1 & 2 & 34 & 5 & 6 & 7 & 8 \\
4 & 3 & 12 & 5 & 6 & 7 & 8
\end{array}\right)=(1432) \\
& \text { (ii) } y=q r=\left(\begin{array}{lllllll}
1 & 2 & 34 & 5 & 6 & 7 & 8 \\
7 & 8 & 56 & 1 & 2 & 3 & 4
\end{array}\right)=(1735)(2846) \\
& \text { (iii) } x^{2}=\left(\begin{array}{lllllll}
1 & 2 & 34 & 5 & 6 & 7 & 8 \\
2 & 1 & 43 & 5 & 6 & 7 & 8
\end{array}\right)=(12)(34) \\
& \text { (iv) } y^{2}=\left(\begin{array}{lllllll}
1 & 2 & 34 & 5 & 6 & 7 & 8 \\
3 & 4 & 12 & 7 & 8 & 5 & 6
\end{array}\right)=(13)(24)(57)(68) \\
& \text { (v) } x y=\left(\begin{array}{lllllll}
1 & 2 & 34 & 5 & 6 & 7 & 8 \\
6 & 5 & 78 & 1 & 2 & 3 & 4
\end{array}\right)=(1625)(37)(48) \\
& \text { (vi) }(x y)^{2}=\left(\begin{array}{lllllll}
1 & 2 & 34 & 5 & 6 & 7 & 8 \\
2 & 1 & 34 & 6 & 5 & 7 & 8
\end{array}\right)=(12)(56) \\
& \text { (vii) } x y^{2}=\left(\begin{array}{lllllll}
1 & 2 & 34 & 5 & 6 & 7 & 8 \\
2 & 1 & 34 & 7 & 8 & 5 & 6
\end{array}\right)=(12)(57)(68) \\
& \text { (viii) } x y^{-1}=\left(\begin{array}{lllllll}
1 & 2 & 34 & 5 & 6 & 7 & 8 \\
8 & 7 & 56 & 3 & 4 & 1 & 2
\end{array}\right)=(1827)(35)(46) \\
& \text { (ix) } y^{-1}=y^{3}=\left(\begin{array}{lllllll}
1 & 2 & 34 & 5 & 6 & 7 & 8 \\
5 & 6 & 78 & 3 & 4 & 1 & 2
\end{array}\right)=(1537)(2648) \\
& \text { (x) } x^{-1}=\left(\begin{array}{lllllll}
1 & 2 & 34 & 5 & 6 & 7 & 8 \\
3 & 4 & 21 & 5 & 6 & 7 & 8
\end{array}\right)=(1324) \\
& \text { (xi) }[x, y]=x^{-1} y^{-1} x y=\left(\begin{array}{lllllll}
1 & 2 & 34 & 5 & 6 & 7 & 8 \\
3 & 4 & 21 & 7 & 8 & 6 & 5
\end{array}\right)=(1324)(5768) \\
& \text { (xii) }[x, y]^{2}=\left(\begin{array}{lllllll}
1 & 2 & 34 & 5 & 6 & 7 & 8 \\
2 & 1 & 43 & 6 & 5 & 8 & 7
\end{array}\right)=(12)(34)(56)(78) \\
& \text { (xiii) } x[x, y]^{2}=\left(\begin{array}{lllllll}
1 & 2 & 34 & 5 & 6 & 7 & 8 \\
3 & 4 & 21 & 6 & 5 & 8 & 7
\end{array}\right)=(1324)(56)(78) \text {. }
\end{aligned}
$$


Using (i) to (ii) the conjugacy classes of $G$ with their cycle patterns are;

$$
\begin{aligned}
& x=\left(\begin{array}{lllllll}
1 & 2 & 34 & 5 & 6 & 7 & 8 \\
4 & 3 & 12 & 5 & 6 & 7 & 8
\end{array}\right), \\
& y=\left(\begin{array}{lllllll}
1 & 2 & 34 & 5 & 6 & 7 & 8 \\
7 & 8 & 56 & 1 & 2 & 3 & 4
\end{array}\right), \\
& x^{2}=\left(\begin{array}{lllllll}
1 & 2 & 34 & 5 & 6 & 7 & 8 \\
2 & 1 & 43 & 5 & 6 & 7 & 8
\end{array}\right), \\
& y^{2}=\left(\begin{array}{lllllll}
1 & 2 & 34 & 5 & 6 & 7 & 8 \\
3 & 4 & 12 & 7 & 8 & 5 & 6
\end{array}\right), \\
& x y=\left(\begin{array}{lllllll}
1 & 2 & 34 & 5 & 6 & 7 & 8 \\
6 & 5 & 78 & 1 & 2 & 3 & 4
\end{array}\right), \\
& (x y)^{2}=\left(\begin{array}{lllllll}
1 & 2 & 34 & 5 & 6 & 7 & 8 \\
2 & 1 & 34 & 6 & 5 & 7 & 8
\end{array}\right) \\
& x y^{2}=\left(\begin{array}{lllllll}
1 & 2 & 34 & 5 & 6 & 7 & 8 \\
2 & 1 & 34 & 7 & 8 & 5 & 6
\end{array}\right), \\
& x y^{3}=x y^{-1}\left(\begin{array}{lllllll}
1 & 2 & 34 & 5 & 6 & 7 & 8 \\
8 & 7 & 56 & 3 & 4 & 1 & 2
\end{array}\right), \\
& {[x, y]=x^{-1} y^{-1} x y=\left(\begin{array}{lllllll}
1 & 2 & 34 & 5 & 6 & 7 & 8 \\
3 & 4 & 21 & 7 & 8 & 6 & 5
\end{array}\right),} \\
& {[x, y]^{2}=\left(\begin{array}{lllllll}
1 & 2 & 34 & 5 & 6 & 7 & 8 \\
2 & 1 & 43 & 6 & 5 & 8 & 7
\end{array}\right),} \\
& x[x, y]^{2}=\left(\begin{array}{lllllll}
1 & 2 & 34 & 5 & 6 & 7 & 8 \\
3 & 4 & 21 & 6 & 5 & 8 & 7
\end{array}\right), \\
& y^{-1}=y^{3}=\left(\begin{array}{lllllll}
1 & 2 & 34 & 5 & 6 & 7 & 8 \\
5 & 6 & 78 & 3 & 4 & 1 & 2
\end{array}\right) .
\end{aligned}
$$

3.2. Element in each conjugacy class of group of order $2^{6}$

Let $C$ denote the classes. Then the elements in each class are outlined as follows; 
C1: $e$ where $e$ is the identity element which always an element in every group.

C2: $C l[x, y]^{2}$

$[x, y]^{2}=(12)(34)(56)(78)$

C3: $\operatorname{Cl}\left(x^{2}\right)$

$x^{2}=(12)(34), \quad\left(x^{2}\right)^{y}=(56)(78)$

$C 4: C l([x, y])$

$[x, y]=(1324)(5768), \quad[x, y]^{y}=(1324)(5867)$,

$[x, y]^{y^{2}}=(1423)(5867), \quad[x, y]^{y^{3}}=(1423)(5768)$

C5: $C l(x)$

$x=(1423), \quad x^{y}=(5768), \quad x^{y^{2}}=(1324), \quad x^{y^{3}}=(5867)$

C6: $\operatorname{Cl}\left(x[x, y]^{2}\right)$

$x[x, y]^{2}=(1324)(56)(78),\left(x[x, y]^{2}\right)^{y}=(5867)(12)(34)$,

$\left(x[x, y]^{2}\right)^{y^{2}}=(1423)(56)(78), \quad\left(x[x, y]^{2}\right)^{y^{3}}=(5768)(12)(34)$

$C 7: C l\left(y^{2}\right)$

$y^{2}=(13)(24)(57)(68), \quad\left(y^{2}\right)^{x}=(13)(24)(57)(68)$,

$\left(y^{2}\right)^{x y}=(13)(24)(58)(67), \quad\left(y^{2}\right)^{x^{2} y}=(14)(23)(58)$

$C 8: C l\left((x y)^{2}\right)$

$(x y)^{2}=(12)(56), \quad\left((x y)^{2}\right)^{x}=(34)(56)$,

$\left((x y)^{2}\right)^{y}=(12)(78), \quad\left((x y)^{2}\right)^{y^{2}}=(34)(78)$ 
C9: $C l\left(x y^{2}\right)$

$x y^{2}=(12)(57)(68), \quad\left(x y^{2}\right)^{x}=(34)(57)(68)$,

$\left(x y^{2}\right)^{y}=(13)(24)(78), \quad\left(x y^{2}\right)^{x y}=(13)(24)(56)$,

$\left(x y^{2}\right)^{y x}=(14)(23)(78), \quad\left(x y^{2}\right)^{y^{2} x}=(34)(58)(67)$,

$\left(x y^{2}\right)^{y^{2} x^{2}}=(12)(58)(67), \quad\left(x y^{2}\right)^{y^{3} x}=(14)(23)(56)$

\section{C10: $C l(y)$}

$y=(1735)(2846), \quad y^{x}=(1547)(2638)$,

$y^{x^{2}}=(1836)(2745), \quad y^{x^{3}}=(1648)(2537)$,

$y^{x y}=(1637)(2548), \quad y^{x^{2} y}=(1746)(2835)$,

$y^{x^{2} y^{2}}=(1845)(2736), \quad y^{x^{3} y}=(1538)(2647)$

\section{C11: $C l\left(y^{-1}\right)$}

$y^{-1}=(1537)(2648), \quad\left(y^{-1}\right)^{x}=(1745)(2836)$,

$\left(y^{-1}\right)^{x^{2}}=(1638)(2547), \quad\left(y^{-1}\right)^{x^{3}}=(1846)(2735)$,

$\left(y^{-1}\right)^{x y}=(1736)(2845), \quad\left(y^{-1}\right)^{x^{3} y}=(1835)(2746)$,

$\left(y^{-1}\right)^{x^{2} y}=(1647)(2538), \quad\left(y^{-1}\right)^{x^{2} y^{2}}=(1548)(2637)$

\section{C12: $C l(x y)$}

$x y=(1625)(37)(48), \quad(x y)^{x}=(3546)(17)(48)$,

$(x y)^{x^{2}}=(1526)(38)(47), \quad(x y)^{x^{3}}=(3645)(18)(27)$,

$(x y)^{y}=(17278)(35)(46), \quad(x y)^{y^{2}}=(3847)(15)(26)$, 


$$
\begin{aligned}
& (x y)^{x^{2} y}=(1827)(36)(45), \quad(x y)^{y x^{3}}=(3748)(16)(25) \\
& C 13: C l\left(x y^{-1}\right) \\
& x y^{-1}=(1827)(35)(46), \quad\left(x y^{-1}\right)^{x}=(3748)(15)(26), \\
& \left(x y^{-1}\right)^{x^{2}}=(1728)(36)(45), \quad\left(x y^{-1}\right)^{x^{3}}=(3847)(16)(25), \\
& \left(x y^{-1}\right)^{x y}=(3645)(17)(28), \quad\left(x y^{-1}\right)^{x^{3} y}=(3546)(18)(27), \\
& \left(x y^{-1}\right)^{x y^{2}}=(1526)(37)(48), \quad\left(x y^{-1}\right)^{x^{2} y}=(1625)(38)(45) .
\end{aligned}
$$

Table 1. Number of elements in each conjugacy class

\begin{tabular}{|c|c|c|}
\hline $\mathrm{S} / \mathrm{N}$ & Classes & Number of elements \\
\hline 1 & $C 1$ & 1 \\
\hline 2 & $C 2$ & 2 \\
\hline 3 & $C 3$ & 4 \\
\hline 4 & $C 4$ & 4 \\
\hline 5 & $C 5$ & 4 \\
\hline 6 & $C 6$ & 4 \\
\hline 7 & $C 7$ & 4 \\
\hline 8 & $C 8$ & 8 \\
\hline 9 & $C 9$ & 8 \\
\hline 10 & $C 10$ & 8 \\
\hline 11 & $C 11$ & 8 \\
\hline 12 & $C 12$ & 8 \\
\hline 13 & $C 13$ & 13 \\
\hline
\end{tabular}

From the enumeration above, a summary of conjugacy class is shown. The number of elements in each class was obtained by counting the number of disjoint cycles form by each class. In Table 1, there are thirteen conjugacy classes of group of order $2^{6}$ and hence thirteen irreducible representations (characters). That is, the number of irreducible representations is equal to the number of conjugacy classes. 


\section{Conclusion}

It has been shown that the number of conjugacy classes of group of order $2^{6}$ can be obtained using the commutator group.

\section{Acknowledgement}

We wish to thank all our lecturers at various levels of our academic pursuit, especially those who taught us group theory. In a special way, the effort of Dr. Victoria Inyang of blessed memory, who supervised the lead author's undergraduate project in 1996 at University of Calabar, Nigeria, in Non-Abelian group is hereby acknowledged with gratitude.

\section{References}

[1] Attila Maróti, Bounding the number of conjugacy classes of a permutation group, School of Mathematics and Statistics, University of Birmingham, UK, 2004, pp. 1-12. http://citeseerx.ist.psu.edu/viewdoc/download?doi=10.1.1.584.4714\&rep=rep1\&type=pdf

[2] L. G. Kovacs and G. R. Robinson, On the number of conjugacy classes of a finite group, Journal of Algebra 160(2) (1993), 441-460. https://doi.org/10.1006/jabr.1993.1196

[3] I. J. Ugbene, E. O. Eze and S. O. Makanjuola, On the number of conjugacy classes in the injective order decreasing transformation semigroup, Pacific Journal of Science and Technology 14(1) (2013), 182-186.

[4] A. Mann, Conjugacy classes in finite groups, Israel Journal of Mathematics 31 (1978), 78-84. https://doi.org/10.1007/BF02761381

[5] A. V. Lopez, Conjugacy classes in finite solvable groups, Israel Journal of Mathematics 47 (1984), 241-245. https://doi.org/10.1007/BF02760519

[6] C. C. Cadogen, On partly ordered partition of a positive integer, Fibonacci Quart. 9(3) (1971), 329-336.

[7] T. W. Judson, Abstract Algebra: Theory and Application, Stephen F. Austin State University, 2010. 